\title{
Residual Stress Influence on the Flexural Buckling of Welded I-Girders
}

\author{
Benjamin Launert ${ }^{1, \mathrm{a}}{ }^{*}$, Michael Rhode ${ }^{2, \mathrm{~b}}$, Arne Kromm $^{2, \mathrm{c}}$, Hartmut Pasternak ${ }^{1, \mathrm{~d}}$ \\ and Thomas Kannengiesser, ${ }^{2, e}$ \\ ${ }^{1}$ Chair of Steel and Timber Structures, Brandenburg University of Technology (BTU), \\ Konrad-Wachsmann-Allee 2, 03046 Cottbus, Germany \\ ${ }^{2}$ Federal Institute for Materials Research and Testing (BAM), Department 9.4 - Weld \\ Mechanics, Unter den Eichen 87, 12205 Berlin, Germany \\ abenjamin.launert@b-tu.de, ’michael.rhode@bam.de, carne.kromm@bam.de, \\ dhartmut.pasternak@b-tu.de, ethomas.kannengiesser@bam.de
}

\begin{abstract}
Keywords: Stability Design, Finite Element Method, 2-D Welding Simulation, Inherent Strain, PDA
\end{abstract}

\begin{abstract}
The nonlinear analysis became a common tool to precisely assess the load-bearing behavior of steel beam and column members. The failure level is significantly influenced by different types of imperfections, among geometric also structural imperfections (residual stresses). Here are still gaps in the knowledge. Nowadays, 3-D welding simulation developed to a level where it could provide reliable estimation of weld-induced distortion and residual stresses. Nevertheless, modelling and computational effort are still in a less practicable range. In this study a simplified procedure to implement residual welding stresses in continuous large scale members is proposed and the influence on the ultimate limit state of slender members in compression is evaluated for two common structural steel grades. The results showed significant improvements in the utilization of load bearing capacity compared with simplified design methods. The comparatively general approach in this study offers potential for future optimization.
\end{abstract}

\section{Introduction}

Welded I-girders are used in different applications in steel construction (e.g. industrial buildings, bridges) due to either dimensions and/or efficiency through customizable plate thicknesses, shapes and/or materials. Many standards, including Eurocode 3 (EC 3) permit the use of non-linear finite element analysis (FEA) for the design of steel structures. The development in this field allows performing "experiments" in computing software instead of the laboratory or expensive in-situ experiments. Still, the implementation of imperfections (including residual stresses due to weld manufacturing) remains a major task in the performance of such analysis. Unlike geometrical imperfections, residual stresses (RS) are not standardized. For that reason, more or less founded simplified distribution functions as proposed by the Swedish design code BSK 99 [1] are used. Limitations on the applicability of this model are not reported except for the plate thicknesses which should not exceed $40 \mathrm{~mm}$. However, the influence of RS seemed somewhat overestimated for many cases comparing conventional structural steel S355 and S690QL. As in the EC 3, direct proportionality of the tensile and compressive RS (the latter are the main interest in this study) and the yield strength is assumed. An opposite effect was recently noticed in [2,3] showing relatively reduced RS in high strength steel. A significant overestimation of RS was generally noticed if the chords are narrow. A lower limit is missing. Additionally, the correlation with the plate thicknesses only is doubted since no record is taken of actual welding parameters. Hence, a new approach was required. This contribution (as part of a national research project [2]) presents an improved general 
procedure to access the load-bearing capacity numerically under realistic consideration of welding effects (with the focus on RS).

\section{2-D Thermo-elasto-plastic analysis}

The geometry is shown in Fig. 1 with the chord width $\left(b_{f}\right) 150 \mathrm{~mm}$ and the web height $\left(h_{w}\right) 220 \mathrm{~mm}$. Plate thicknesses were $25 \mathrm{~mm}\left(\mathrm{t}_{\mathrm{f}}\right)$ and $15 \mathrm{~mm}\left(\mathrm{t}_{\mathrm{w}}\right)$ and base materials were conventional S355 and S690QL. The weld type is a T-joint with subsequently welded two-sided single-layer fillets. A reference model (3-D simulation) was presented in [3]. The calculation time was several days. Thus, a simplified modelling procedure is absolutely required in practice. A simplified 2-D modelling procedure in Ansys (16.2) was developed. The results are shown Fig. 1. Verification is presented in Fig. 2.

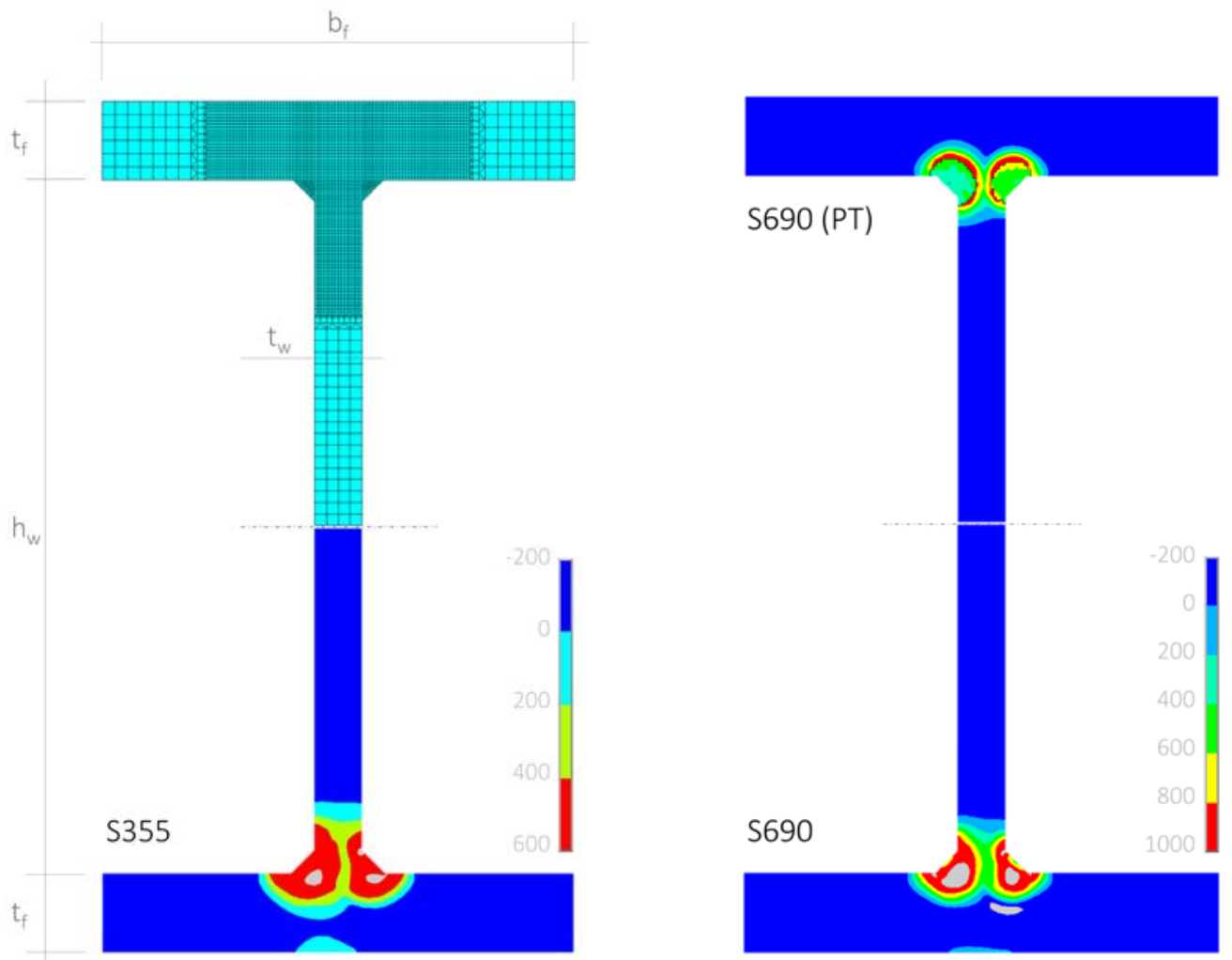

Fig. 1: Meshed 2-D model and calculated RS (in MPa) for S355 and S690QL (with/without PT)
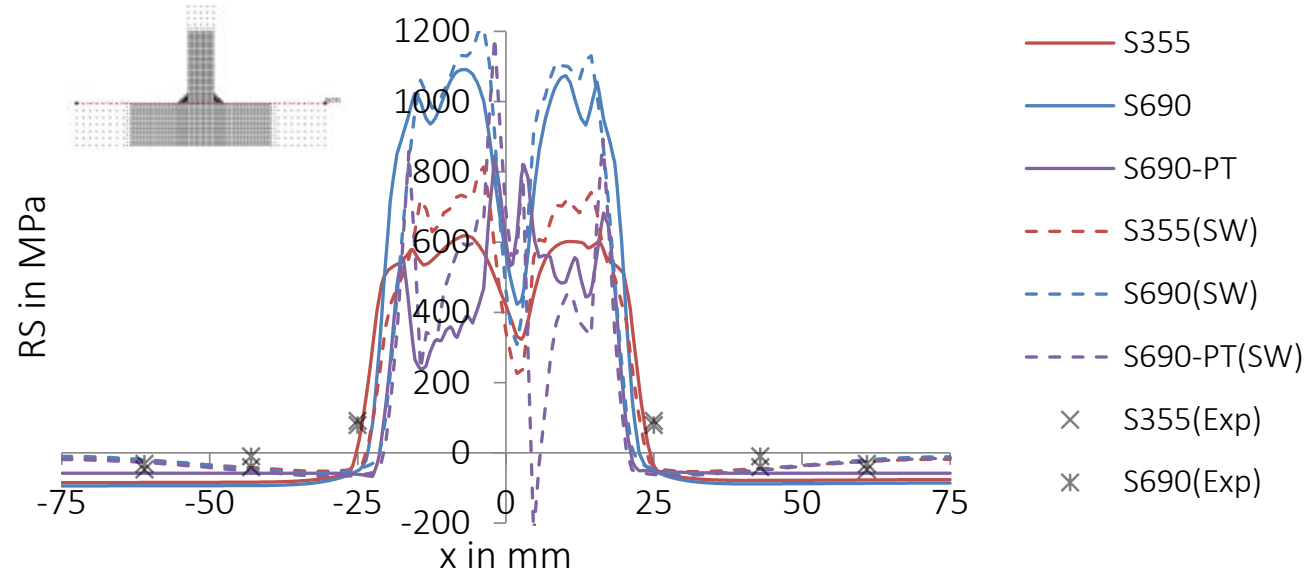

Fig. 2: RS distribution compared to [3], SW-Simufact, Exp-Experiment, exemplary for the chord 
Elements types were plane 55 for the thermal analysis and plane 182 for the mechanics. A generalized plane strain condition was assumed. The element edge length close to the weld was 1 mm. A model using symmetry condition about the web middle axis has shown identical stresses compared with those determined from the full section model (which is due to the web height in this example). Nevertheless, the computation effort was comparatively small also for the full section model (less than half an hour). The temperatures were more or less independent of the grade and mostly depend on welding parameters. The particular yield strengths (mean values were 463,9 $\mathrm{MPa}$ for S355 and 831,3 MPa for S690QL) were used for scaling of the stress strain behaviour. The hardening behaviour was simplified (a constant hardening modulus of $1000 \mathrm{MPa}$ was assumed at all temperature levels). Further data (Young's modulus, Poisson ratio and thermal expansion coefficient) were taken as done in [3]. A good match of (longitudinal) RS was achieved. The peaks are slightly underestimated. An additional simplified modelling procedure (PT) was used for consideration of transformation effects during heating and cooling in S690QL. The results were comparable as well even with their simplifications.

\section{Plasticity-based analysis}

The following part is based on the assumption that RS are caused by incompatible permanent strains (so called inherent strains [4,5]) induced by either temperature gradients and/or phase transformations. This analysis is therefore sometimes referred as plasticity-based analysis (PDA) [6]. The actual analysis is of elastic type though. The procedure is that initial strains are applied to the full-structure FE-model and that "reactions" in terms of deformations and stresses are calculated by conducting a linear elastic analysis. Inherent strain (taken as the accumulated plastic strain) in a weld joint is produced only in a limited region near the weld. However, RS (or elastic strain) due to inherent strain is produced in the entire weldment. Their relations are expressed by elastic response equations. This is the idea behind PDA.

Applications were carried out mainly to control deformations in large welded structures using equivalent forces and moments (known as Equivalent Load Method). Numerical procedures incorporating inherent strain directly are limited. The coupling with a subsequent capacity analysis is of interest in structural design but has not been realized either. The plastic strain distribution can be calculated easily using simplified 2-D modelling approach. The distribution in T-joints obtained from comparative 2-D and 3-D models was however found quite different except of those associated with the longitudinal direction [6]. Thus, 2-D model may not be applicable in the prediction of angular distortion in $\mathrm{T}$-joints but in the prediction of longitudinal RS and longitudinal buckling which strongly depends upon the distribution of longitudinal cumulative plastic strain [6]. A direct transfer (as proposed in [6]) seemed unnecessary. A modified procedure is presented and detailed in subsequent paragraphs.

1. 2-D thermal elastic-plastic analysis (TEPA) to calculate the distribution of (longitudinal) cumulative plastic strain,

2. Definition of a simplified distribution pattern,

3. Creation of a suitable mesh applicable to the criteria of PDA and capacity analysis and

4. Elastic analysis of the full-structure elastic FE-model imposing initial strain to reproduce RS (and deformations).

The distribution of plastic strain is initially needed from TEPA (1.). This is shown in Fig. 3 a) in case of S690. It was noticed that the plastic strain pattern is typically of somewhat simple shape. For that reason, a simplified distribution pattern was derived (2.). According to [5], the inherent strain zone of a single fillet weld may be generally simplified by three partial ellipses and the fillet weld metal. This approach was simplified to ensure a better applicability of the model. An ellipsoid (radii are indicated by $r_{x}$ and $r_{y}$ ) with the center in the application point of the weld torch was used (see Fig. 3 a) as well). From the distribution function it is known that the maximum values are 
approximately equal to the yield strain $\left(\varepsilon_{\mathrm{y}}\right)$ within the first ellipsoid. Values then drop linearly to zero to the border of the second ellipsoid. Elsewhere no initial strains are applied. A section of the distribution at the top surface of the chord is given in comparison with reference data in Fig. 3 b). A reasonable agreement was observed.
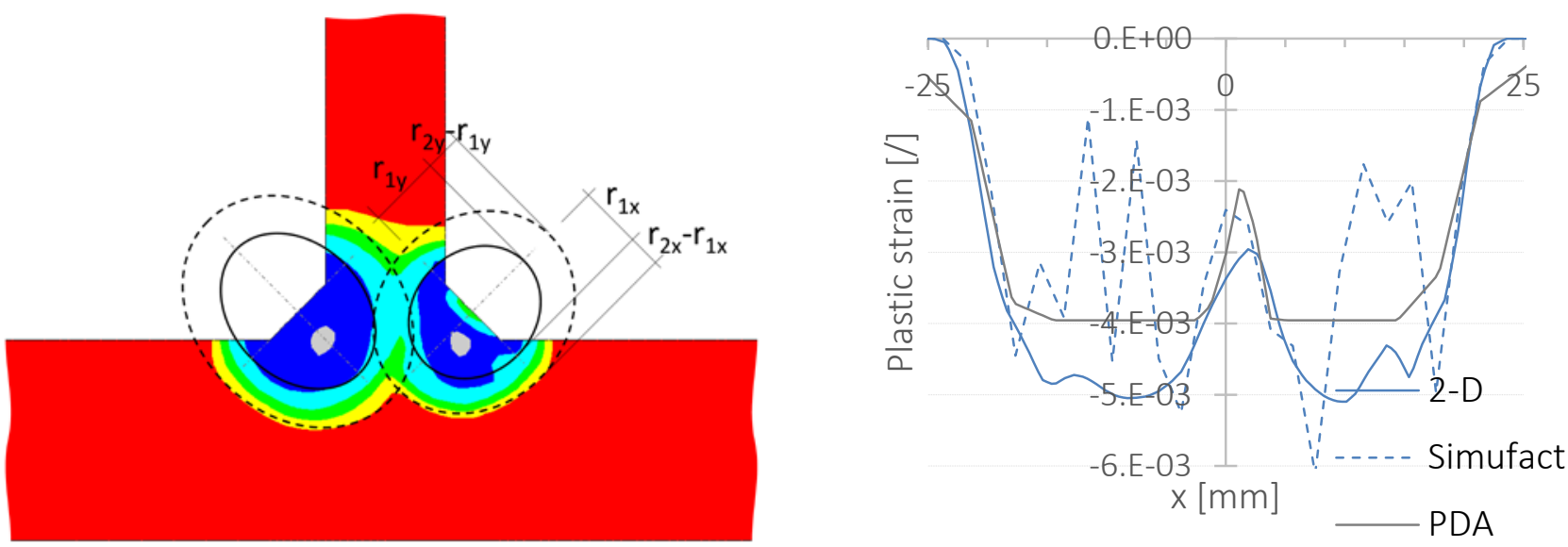

Fig. 3: Calculated plastic strain distribution compared to proposed simplified method (exemplary for $S 690 Q L$ )

The implementation in the global (3-D) FE-model was realized using the "inistate" command (alternative ways are possible, e.g. by the definition of artificial expansion coefficients to create equivalent thermal strains as proposed in [6]). A certain restriction is that PDA requires a fine mesh to ensure that initial strains are applied precisely. It is therefore necessary to provide a suitable mesh applicable to the criteria of PDA and capacity analysis (3.). This is, to a certain degree, always a compromise. An adapted 2-D mesh was generated with a reduction in element numbers from 6480 to 1092. This mesh was extruded in longitudinal direction (to different length) with element lengths of $15 \mathrm{~mm}$. The element type has then been changed to solid 185. Finally, a linear elastic analysis was conducted (4.). It was shown that this procedure is capable of creating realistic RS (and also deformations) which can be directly utilized in a subsequent capacity analysis. Applicability in terms of deformations was exemplified but only verified qualitatively yet. Hence, only the RS are used in section 4.

\section{Load bearing capacity}

The final step was a nonlinear analysis of the load-bearing behaviour. The static system is a simply supported beam under pure compression. The investigations are given exemplary for weak-axis buckling. The cross section is the same as given in section 2 . The material behaviour is equal to the one from the TEPA except that values are only needed for room temperature. Deviations from the ideal shape (so called geometrical imperfections) were taken as the first eigenmode determined from linear buckling analysis (LBA). The maximum value was scaled to L/1000 (which is the maximum permitted manufacturing tolerance). This is a conservative assumption, but represents the state-of-art. The PDA could be used for future deformation analysis. For the consideration of RS, three approaches were compared:

Case 1) Without $R S$,

Case 2) Direct definition of RS based on BSK 99 [1] and

Case 3) Indirect generation of RS using PDA (definition of initial strains by inistate). 
EC 3 and other standards use buckling curves. Load influencing factors are covered by the definition of so called reduction factors $\chi$. Depending on section type, manufacture, failure and/or direction of failure and in parts material, different buckling curves are distinguished. The reduction factor $\chi$ is given by Eq. 1

$$
\chi=\frac{N_{R}}{N_{p l}}
$$

The slenderness $\bar{\lambda}$ is varied by the length $l$ and is given by Eq. 2

$$
\begin{aligned}
& \bar{\lambda}=\sqrt{\frac{N_{p l}}{N_{c r}}} \quad, \text { where } \\
& N_{p l}=A \cdot f_{y}, N_{c r}=\frac{\pi^{2} \cdot E \cdot I}{l^{2}} .
\end{aligned}
$$

Accordingly, the length $l$ to be used in the analysis for certain slenderness $\bar{\lambda}$ can be given by Eq. 3

$$
l=\sqrt{\bar{\lambda}^{2} \cdot \frac{\pi^{2} \cdot E \cdot I}{A \cdot f_{y}} .}
$$

The lengths $l$ were calculated using the cross-sectional properties without consideration of the weld seams. The slenderness range relevant for RS was found close to 1,0 [7]. For that reason, the slenderness $\bar{\lambda}$ was varied in steps of 0,2 from 0,6 to 1,4 . Solid element type 185 was used. As for the welding, this was necessary due to the local plastic strain distribution for case 3$)$. Works are ongoing to make the method less sensitive to the mesh and to reduce the element level in terms of shell (or even beam) element types suitable in this context. Solid model was not necessary for cases 1) and 2), but has been used for comparative purpose. The calculated results are given in Fig. 4. The values are compared with the buckling curves stated by the EC 3. For the investigated case, curve "c" applies for comparison.
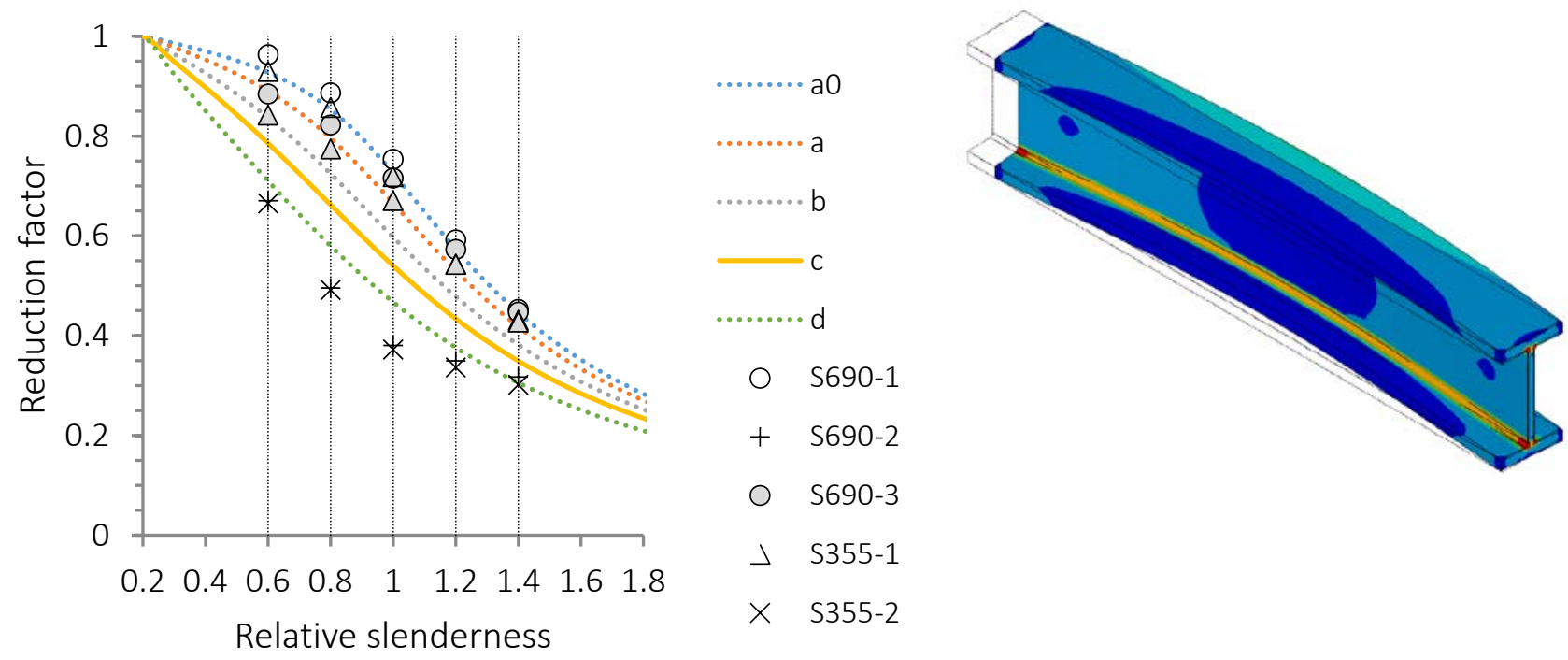

Fig. 4: Results in comparison with European buckling curves (EC 3) 
The comparison of results without RS shows that there are minor differences with an increasing yield strength difference though values are normalized. With RS, results differ significantly. The RS model by [1] led to highly unrealistic compressive RS (see Introduction) causing a significant underestimation of the ultimate load. Instead, the RS generated through inherent strain (PDA) equalled realistic RS. The comparison of materials shows generally favourable behavior of the S690, which is explained by relatively decreased compressive RS. On average, the buckling curve is raised by one line indicated by curve "b" for the S355 and curve "a" for the S690. With respect to EC 3, large reserves are identified.

\section{Summary}

An improved method for the capacity accessment of welded girders was presented. The modelling using 3-D solid elements in welding simulation is often not required in case of constant cross sections through the lengths. Nearly identical results are generated using 2-D solid models encompassing the assumption of generalized plane strain. The observed computation times demonstrated the applicability in practice. The calculated plastic strain distribution was then simplified for application to the total girder. A first approach was presented using two ellipsoids for each fillet. This resulted in good agreement with reference data. A further standardization of these variables could be helpful. The method is however, to some extent, restricted by the necessary local discretization density, which is needed in similar form also for the capacity model. The transferability to analogeous shell or beam models requires further research. Nevertheless, the proposed method is a significant improvement in comparison with existent simplified models. A particular advantage is the wide applicability apart from the herein presented example (easy adaption to different cross sectional shapes). The method appeared principally also suitable to take into account weld-induced deformation of large structures. The capacity analysis was eventually on an example for weak-axis buckling of a centrally loaded compression member. Results showed the superior buckling behavior of high strength steel S690. The assignment of a buckling curve was approximately one curve higher compared to that of conventional S355.

\section{References}

[1] N.N., Swedish Design Rules for Steel Structures, Boverket - Swedish National Board of Housing, Building and Planning, Karlskrona, 2003.

[2] H. Pasternak, T. Kannengiesser et al., IGF 18104 BG - Enhancement of Load Capacity of Welded High-Strength I-Shape Sections Using Improved Design Models for the Consideration of Residual Stress (in German), 04/2014-04/2016, Project description, 2013.

[3] H. Pasternak, B. Launert, T. Kannengiesser and M. Rhode, Advanced residual stress assessment of plate girders through welding simulation, submitted to Procedia Engineering (2016), $12^{\text {th }}$

International Conference "Modern Building Materials, Structures and Techniques", May $26^{\text {th }}-27^{\text {th }}$, 2016, Vilnius.

[4] Y. Ueda, M. G. Yuan, The Characteristics of the Source of Welding Residual Stress (Inherent Strain) and Its Application to Measurement and Prediction, Trans. JWRI 20 (1991), No. 2, 119-127. [5] Y. Ueda, N.-X. Ma, Measuring Methods of Three-Dimensional Residual Stresses with Aid of Distribution Function of Inherent Strains (Report 3): Distributions of Residual stresses and Inherent Strains in Fillet Welds, Trans. JWRI 24 (1995), No. 2, 123-130.

[6] G. Jung, Plasticity based distortion analysis for fillet welded thin plate t-joints, PhD-thesis, Ohio State University, 2003.

[7] H. Pasternak, B. Launert and T. Krausche, Welding of girders with thick plates - Fabrication, measurement and simulation, Journal of Constructional Steel Research 115 (2015), 407-416.

http://dx.doi.org/10.1016/j.jcsr.2015.08.037 